\title{
Solution NMR Structure of a Ligand/Hybrid-2-G-Quadruplex Complex Reveals Rearrangements that Affect Ligand Binding
}

\author{
Julia Wirmer-Bartoschek, Lars Erik Bendel, Hendrik R. A. Jonker, J. Tassilo Grün, \\ Francesco Papi, Carla Bazzicalupi, Luigi Messori, Paola Gratteri,* and Harald Schwalbe*
}

\begin{abstract}
Telomeric G-quadruplexes have recently emerged as drug targets in cancer research. Herein, we present the first $N M R$ structure of a telomeric DNA G-quadruplex that adopts the biologically relevant hybrid-2 conformation in a ligandbound state. We solved the complex with a metalorganic gold(III) ligand that stabilizes G-quadruplexes. Analysis of the free and bound structures reveals structural changes in the capping region of the G-quadruplex. The ligand is sandwiched between one terminal G-tetrad and a flanking nucleotide. This complex structure involves a major structural rearrangement compared to the free G-quadruplex structure as observed for other $G$-quadruplexes in different conformations, invalidating simple docking approaches to ligand-G-quadruplex structure determination.
\end{abstract}

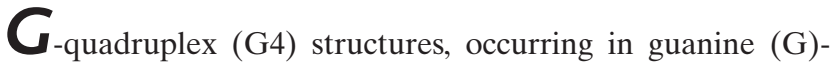
rich DNA and RNA sequences, have recently emerged as important targets for drugs in cancer research. ${ }^{[1]}$ G-rich sequences are found in telomeres ${ }^{[2]}$ and in promoter regions particularly of cancer genes, ${ }^{[3]}$ as first shown for the $M Y C$ oncogene. ${ }^{[4]}$

The human telomeric DNA is 5-8kb long and consists of d(TTAGGG) $)_{n}$ tandem repeats. ${ }^{[5]}$ The $3^{\prime}$ end of the telomeric DNA consists of 100-200 nt single-stranded DNA overhangs of these tandem repeats. The overhangs of telomeric DNA are shortened during each cell division and this shortening leads to cell aging and death. In cancer cells, this mechanism is not operational, because the telomerase that maintains DNA length is upregulated in $80-90 \%$ of cancer cells. ${ }^{[6]}$

Stabilization of G-quadruplex structures is thought to repress the lengthening of telomers by the telomerase.

[*] Dr. J. Wirmer-Bartoschek, L. E. Bendel, Dr. H. R. A. Jonker, J. T. Grün, Prof. Dr. H. Schwalbe

Institute for Organic Chemistry and Chemical Biology, Center of Biomolecular Magnetic Resonance (BMRZ), Goethe University Frankfurt/Main

Max-von-Laue-Strasse 7, 60439 Frankfurt (Germany)

E-mail: schwalbe@nmr.uni-frankfurt.de

Dr. F. Papi, Prof. Dr. C. Bazzicalupi, Prof. Dr. L. Messori Dipartimento di Chimica "Ugo Schiff"

Università degli Studi di Firenze

Via della Lastruccia 3, 50019 Sesto Fiorentino (FI) (Italy)

Prof. Dr. P. Gratteri

Dipartimento di Neuroscienze, Psicologia, Area del Farmaco, Salute del Bambino (NEUROFARBA), Università degli Studi di Firenze via Ugo Schiff 6, 50019 Sesto Fiorentino (Italy)

E-mail: paola.gratteri@unifi.it

(9) Supporting information for this article can be found under: https://doi.org/10.1002/anie.201702135.
However, the exact mechanism has remained elusive. In 1991 it was shown, that the formation of G-quadruplex structures inhibits the function of the Oxytricha nova telomerase in vitro. ${ }^{[7]}$ Telomer elongation is further promoted by complex formation of protein POT1 as part of the shelterin complex and the telomerase complex. ${ }^{[8]}$ In this, binding of POT1 destabilizes G-quadruplexes allowing telomerase extension in vitro. ${ }^{[9]}$

Stabilization of G4 structures is thus important. However, targeting G4 structures by small molecules is challenging, partly because of G4 structural polymorphism: ${ }^{[10]}$ Depending on the exact length and sequence of overhanging nucleotides as well as salt and solvent conditions, different conformations of the G4 are observed. ${ }^{[11]}$ Currently, evidence is accumulating that hybrid and 2-tetrads basket conformations are adopted under physiological conditions. ${ }^{[12]}$ Both, hybrid-1 and hybrid-2 apo structures were elucidated in $2007,{ }^{[13]}$ revealing capping of the outer tetrads by flanking and loop residues. By contrast, ligand-bound structures of G-quadruplexes adopting the physiological relevant hybrid-2 conformation have not been reported yet. Ligand-bound structures of other G4 polymorphs show conformational rearrangements aimed to accommodate the ligand, for example in the cmyc G4 and in the hybrid-1 G4 of telomeric DNA. ${ }^{[14]}$ It follows that the application of a simple, rigid docking approach utilizing the conformation of the free state as the conformation adopted by the G4 DNA in the ligand-bound state is therefore not appropriate. Instead, molecular dynamic of induced-fit simulation procedures should be considered as useful investigation tools. Indeed, it is crucial to obtain exact structural information on the bound state of the hybrid-2 G4, which appears to be a physiological relevant conformation.

We report herein the first de novo structure of a telomeric G-quadruplex-ligand complex in a hybrid-2 conformation solved by NMR spectroscopy. The ligand is a binuclear gold(III)-complex (Auoxo6) consisting of two bipyridyl gold(III) moieties connected through a dioxo bridge as depicted in Figure 1. The compound has previously been validated in its potential role as drug lead and exhibits cytotoxic effects in a variety of cancer cell lines, with $\mathrm{IC}_{50}$ values in the low micromolar range. ${ }^{[15]}$ Auoxo6 inhibits the enzyme thioredoxin reductase, which plays a crucial role in governing redox metabolism. ${ }^{[16]}$ Proteomic studies further revealed that Auoxo6 affects the intracellular redox metabolism as well as the proteasome system. ${ }^{[17]}$ It was previously shown that Auoxo6 binds to G4 DNAs and selectively stabilizes these non-canonical DNA structures, while double stranded DNA is not stabilized against thermal denaturation. ${ }^{[18]}$ These results strongly suggest quadruplex DNA as a molecular target for 
a)

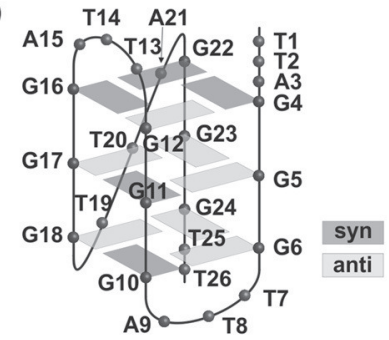

c)

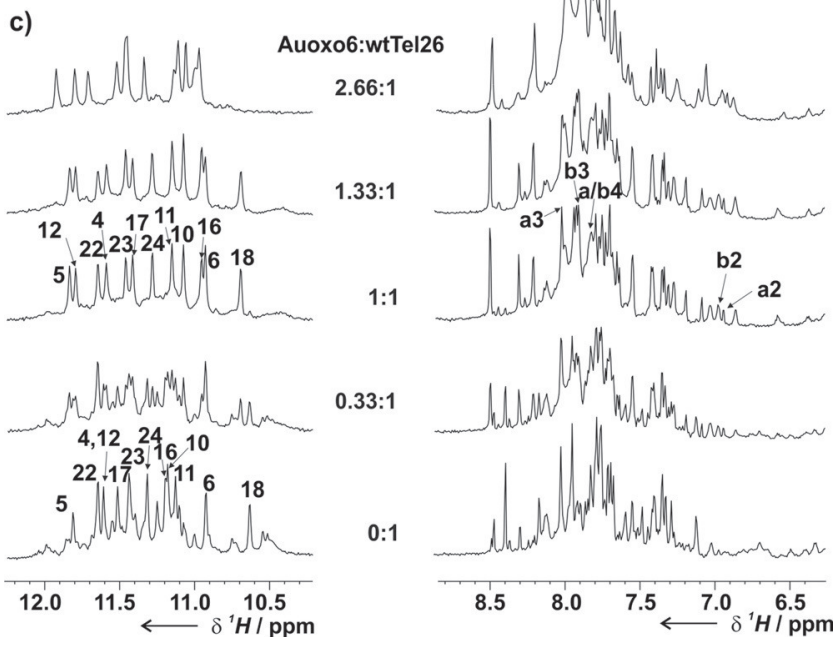

Figure 1. a) Configuration of the investigated DNA quadruplex (hybrid2). b) Auoxo6, $r 1$ to $r 4$ denote the aromatic rings, $a$ and $b$ denote different spin systems observed in the NMR spectra, numbering of aromatic protons is given. c) NMR imino and aromatic proton spectral regions. Titration of Auoxo6 in the presence of wtTel26 (5'.

(TTACGG) 4 TT- $3^{\prime}$ ) with site resolution in the imino region. The titration was recorded at $700 \mathrm{MHz}, 298 \mathrm{~K}$, at a concentration of $0.1 \mathrm{~mm}$ DNA, $70 \mathrm{~mm} \mathrm{KCl}, 25 \mathrm{~mm} \mathrm{KP}$, $\mathrm{pH}$ 7.0. The imino assignment of free DNA taken from Ref. [13a]. The imino assignment of ligand-bound wtTel26 is indicated in the imino region, assignment of aromatic ligand protons is given in the aromatic region.

Auoxo6. Conclusive evidence, however, still needs to be provided.

First, we investigated the binding of Auoxo6 to wtTel26 DNA (5'(TTAGGG) ${ }_{4}$ TT- $\left.3^{\prime}\right)$ by ${ }^{1}$ H-1D NMR spectroscopy (Figure 1). Upon titration of DNA with Auoxo6, a second set of signals appears at substoichiometric concentration of the ligand, well visible in the imino region, while the aromatic region is crowded. This second set of signals is in slow exchange with the signals of the free DNA. After addition of one equivalent of Auoxo6 at a DNA concentration of $0.1 \mathrm{~mm}$, signals of the free DNA have disappeared. At higher ligand concentrations (2.66:1 ligand/DNA ratio), a third set of signals is observed accounting for more than $90 \%$ total signal intensity. This third set of signals, however, is slightly broadened compared to the 1:1 state and may result from a state with two or more ligands bound to a single DNA molecule. In this work, we focus on the characterization of the 1:1 complex.

In the free state of the DNA, the hybrid-2 structure is the major conformation, populated to approximately $70 \% .{ }^{[13 \mathrm{a}]} \mathrm{In}$ the bound state, the equilibrium is shifted to more than $90 \%$ hybrid-2 conformation.
Binding of Auoxo6 to another G-quadruplex, the Tel26 sequence, which adopts the hybrid-1 conformation, can also be detected (Supporting Information, Figure S1). The ligand binds in slow exchange to the hybrid-1 DNA, however, it is not possible to shift the equilibrium completely towards a single defined, stable bound state; instead, aggregation of the system is observed at ligand:DNA ratios of 2:1 and higher.

For the assignment of the wtTel26:Auoxo6 complex, exchange peaks between the free DNA and the complex, at [Auoxo6]:[DNA] = 0.7:1, were used (Supporting Information, Figure S2). Complete proton assignment of the 1:1 complex was obtained using NOESY, TOCSY, COSY, and natural abundance ${ }^{13} \mathrm{C}$-HSQC spectra at [Auoxo6]:[DNA] $=1.3: 1$ (Supporting Information, Figures S2-S5).

At these concentrations, three ligand spin systems can be observed in the aromatic region of a COSY spectrum (Figure S3), in contrast to the single spin system observed for the $D_{2 h}$-symmetric free ligand. Two signal sets have similar high intensities, while weak residual signals of a low molecular weight ligand free in solution can also be detected. For the strong two sets aromatic resonances in the ${ }^{13} \mathrm{C}-\mathrm{HSQC}$ can also be assigned (Figure S4). Weak ${ }^{4} J$ aromatic proton-methyl crosspeaks for bound sets can be identified in the spectrum, aiding unambiguous assignments. The methyl groups of the bound ligand show NOEs to the imino protons of G4, G12, G16, and G22 in the $5^{\prime}$ tetrad of the quadruplex (Figure 2). The imino protons of G12 and G16 show the largest chemical shift differences $(>0.18 \mathrm{ppm})$. Auoxo6 stacks on top of the terminal tetrad, which represents the common binding mode of aromatic ligands. ${ }^{[19]}$

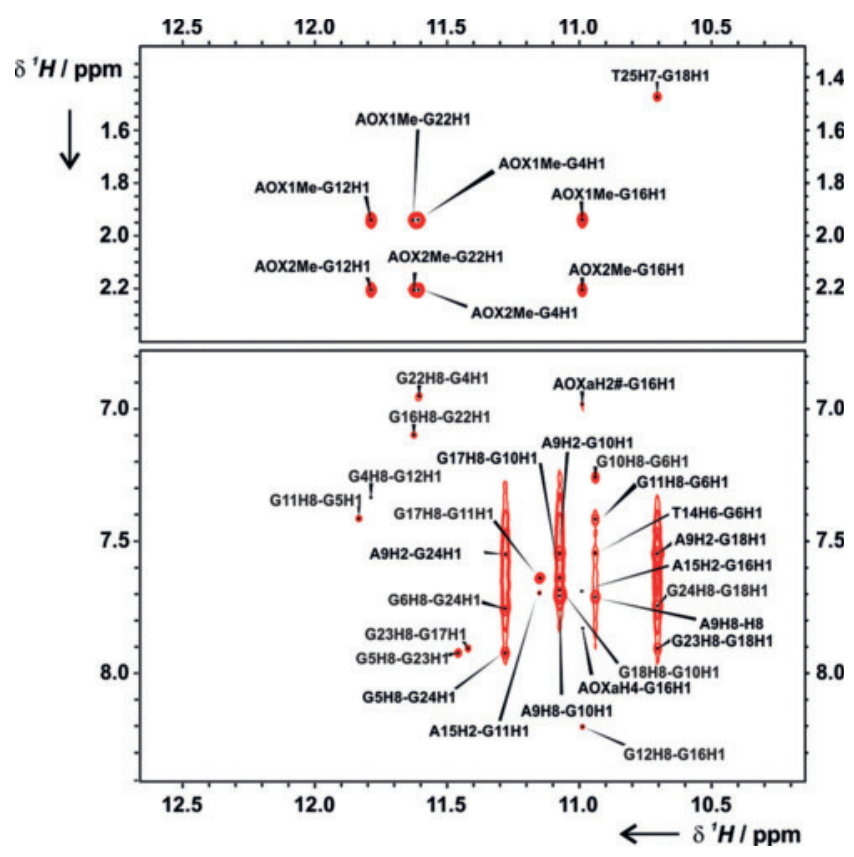

Figure 2. Imino-methyl and imino-aromatic sections of a $2 \mathrm{D}{ }^{1} \mathrm{H},{ }^{1} \mathrm{H}$ NOESY spectrum. Assignment of crosspeaks is indicated, AOXa and AOXb refers to the two signal sets of the ligand. The spectrum was recorded at $700 \mathrm{MHz}, 300 \mathrm{~ms}$ mixing time at $298 \mathrm{~K}$ with concentrations of $1.3 \mathrm{~mm}$ Auoxo6, $1 \mathrm{~mm}$ wtTel26, $70 \mathrm{~mm} \mathrm{KCl}, 25 \mathrm{~mm} \mathrm{KP}$, $\mathrm{pH} 7.0$. 
Further ligand-to-DNA NOEs are observed for residues T2, A3, G4, G12, T13, T14, A15, G16, and G22. A total of 58 NOEs between the ligand and the DNA are observed.

The presence of the two signal sets for the symmetric ligand Auoxo6 suggests different possibilities for ligand binding. The titration (Figure 1) shows saturation at a 1:1 stoichiometry excluding the possibility of a 2:1 (ligand:DNA) complex. Both sets of signals show contacts to the same region of the DNA (excluding two distinct binding sites). These observations leave open two options for ligand binding: 1) The ligand binds in two different binding modes, which are in exchange with each other. The assignment of the rings would be degenerate, each signal set would belong to a different but closely related binding mode. This is however unlikely, given the relative uniform broadening of the imino resonances. It is more conceivable that 2) the four-fold symmetry of the ligand signals is reduced upon binding. In this case, different scenarios are possible for the assignment of the aromatic rings of the ligand: i) Either two of the rings are the same or ii) one ring differs from the other three rings. Due to the intensities of the signals, option (i) is more likely. All possibilities were tested during the structure calculation using Aria and its automated NOE assignment procedure. The results of the structure calculations indicate that the four-fold degeneracy of the rings is broken by the binding of the ligand: The protons of rings 1 and 4 and the protons of rings 2 and 3 resonate at a different position (option $2 \mathrm{i}$ ).

The bundle of the 10 best structures of the wtTel26Auoxo6 complex has an RMSD of $1.8 \AA$ using all atoms and an RMSD of $0.7 \AA$ for the guanine residues only (Table 1 ). While the core of the G4 including the stacking A3 is well defined $(\mathrm{RMSD}=0.9 \AA$ ), the loops and the termini are less well defined.

The DNA adopts the hybrid-2 conformation (Figure 1a) in which strands 1,3 , and 4 are parallel and strand 2 is antiparallel (Figure 3). Strands 3 and 4 are connected by

Table 1: Structural statistics for the ensemble of the 10 best NMR structures.

\begin{tabular}{lll}
\hline NOE distance restraints & & \\
Unambiguous NOEs: & intra-residue & 606 \\
& sequential & 357 \\
& medium range & 158 \\
& long range & 23 \\
& DNA-Ligand & 29 \\
& & 39 \\
Ambiguous NOEs: & intra-residue & 226 \\
& intra-DNA & 9 \\
& DNA or Ligand & 173 \\
& DNA-Ligand & 25 \\
Distance restraints & & 19 \\
& hydrogen bonds & 24 \\
Base planarity & potassium site coordination & 16 \\
Torsion angles & & 12 \\
Violations & & 12 \\
& & \\
RMSD & & 0 \\
\hline
\end{tabular}

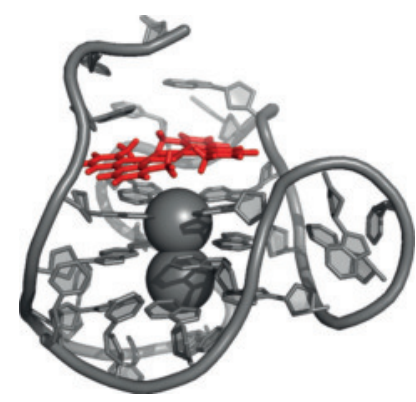

Figure 3. NMR-structural model of the wtTel26 complex (DNA, $\mathrm{K}+$ ions $=$ grey, Auoxo6=red). PDB-code: 5MVB; BMRB-code: 34086.

a double-chain-reversal loop, which covers groove 3. The glycosidic torsion angles within the G-tetrads are syn-antisyn-syn (5'tetrad), anti-syn-anti-anti (middle tetrad) and antisyn-anti-anti (3'tetrad). G24 adopts a C3'-endo sugar pucker, which is confirmed by COSY crosspeak intensities ( $\mathrm{H} 1^{\prime}-\mathrm{H} 2^{\prime}$ l $\mathrm{H} 2^{\prime \prime}$ and $\mathrm{H}^{\prime}-\mathrm{H} 4^{\prime}$ crosspeaks and the observed NOESY crosspeak intensities $\left(\mathrm{H}^{\prime}-\mathrm{H} 2^{\prime} / \mathrm{H} 2^{\prime \prime}\right)$. Except for the terminal nucleotide $\mathrm{T} 1$, all other sugar puckers observed in the calculated structure are in south conformations with pseudorotation angles ${ }^{[20]}$ between 113 and $188^{\circ}$. Detected sugar puckers do not all fall into the range of $\mathrm{C}^{\prime}$ endo sugar pucker observed in B-DNA but show a greater variety, which can be qualitatively seen by the different splittings and intensities in the DQF-COSY (Figure S5). Deviations from the typical C2' endo conformation in DNA has also been observed previously for non-canonical DNA structures. ${ }^{[21]}$

The ligand-binding pocket involving the $5^{\prime}$-capping structure is well defined in the structural ensemble (Supporting Information, Figure S6). The ligand stacks on top of the 5' tetrad. It is not located within the center of the tetrad but shifted towards G4, G12, and G16. For G4, sugar-ligand NOEs are also observed, while for the other three nucleotides only imino-signals from the nucleobases show NOE contacts to the ligand. A3 stacks on the ligand with multiple NOEs of one of the bipyridyl moieties of the ligand to all protons (sugar and nucleobase), except the $\mathrm{H}^{\prime}$ protons of A3. A15 does not stack on the ligand but is oriented by the sugar edge towards the ligand, particularly to the other bipyridyl moiety, which is manifested by a number of NOEs between the $\mathrm{H} 2$ aromatic proton as well as the sugar protons of A15 with the ligand. Few further NOEs to the ligand could be observed from the less-well-defined residues T2 and T13.

A comparison of the complex to the previously published free structure (2JPZ ${ }^{[13 a]}$ reveals a major structural rearrangement in the loop/capping regions of the G-quadruplex upon ligand binding (Figure 4). While the core of the G-quadruplex in the free and bound forms is comparable with an RMSD of $1.3 \AA$ (Supporting Information, Figure S7), the overall RMSD between the bound and the free structures is $3.4 \AA$. In the apo G4, A3, T13, and A15 stack on top of the 5'-tetrad, building a flexible capping structure without detectable hydrogen bonds. This capping structure at the $5^{\prime}$-end of the free G-quadruplex DNA (A3, T13, and A15) is opened up in the bound state to accommodate the ligand, while A3 is shifted upwards compared to the free state and stacks on the ligand. 

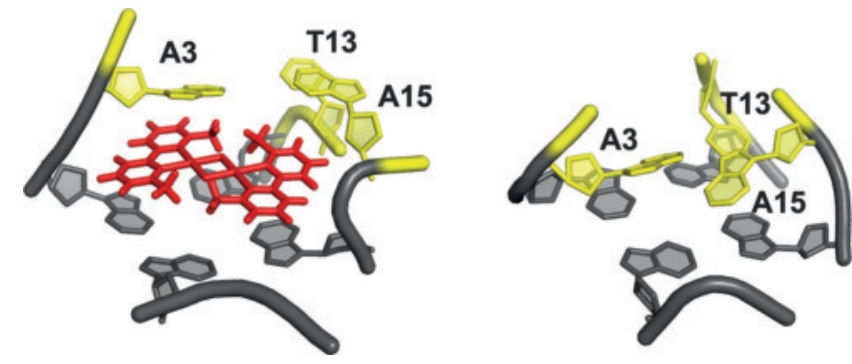

Figure 4. Comparison of the bound and the free state of the DNA. Left: wtTel26-Auoxo6 (pdb: 5MVB); right: free wtTel26 (pdb:2JPZ) ${ }^{[13 a]}$ $\left(5^{\prime}\right.$ tetrad $=$ grey; $A 3, T 13$, and A15=yellow; Auoxo6 $=$ red $)$.

Further differences between the bound and the free structure are observed at the capping site of the $3^{\prime}$ tetrad. A stable capping structure is observed in the NMR-structure of the free state, ${ }^{[13 a]}$ while the respective residues $\mathrm{T} 8, \mathrm{~A} 9$, and T25 are not well defined in our structure. These differences may be due to different temperature conditions of the measured data used. While the free G-quadruplex structure was determined using data obtained at $1-30^{\circ} \mathrm{C}$ with iminosignals of the $3^{\prime}$ capping structure visible at low temperature, our structure is based on data obtained at $25^{\circ} \mathrm{C}$; under these conditions, only guanine imino-signals are visible in the spectrum. The capping structure at the $3^{\prime}$ tetrad is thus not as well defined, suggesting the possibility of opening up to accommodate a second ligand stacking on the $3^{\prime}$ tetrad.

In the free DNA, stacking of the capping nucleotides of the $5^{\prime}$ tetrad is rather weak, with the three nucleotides being non-planar in the free structure. In contrast, the $3^{\prime}$ capping structure is well structured in the free DNA, which is in line with the preferred binding of the ligand to the $5^{\prime}$ tetrad as the capping structure is more flexible. We attribute the lower stability of the Tel26 DNA complex to the higher stability of the $5^{\prime}$ capping structure of this DNA in the free state, compared to the wtTel26 DNA. We therefore propose that flexibility of the capping structure is a general motif for the binding of aromatic ligands to isolated hybrid G4 structures. While we believe that the hybrid- 2 conformation is biological relevant, a clear picture of how sequential G4 structures in the telomeres interact and thereby may alter the binding pocket is not yet available. Obtaining a picture of the binding pocket in the context of higher order G4s therefore remains subject of future studies.

In conclusion, we present the NMR structure determination of the high-affinity complex of a G-quadruplex that binds a dinuclear metal-compound ligand containing two central Au cations. Such gold complexes are potential new anti-cancer agents. The conformational equilibrium of the target DNA has been described, in which the equilibrium between hybrid-2 folding and minor conformers in the free DNA is shifted towards the hybrid- 2 conformation, the ligand therefore shows a modest degree of conformational selection differentiating between DNA quadruplex polymorphs. Binding involves a conformational rearrangement of the $5^{\prime}$ capping moiety of the $\mathrm{G} 4$, invalidating rigid-body docking approaches on the unbound target to deduce the complex structure. Our structure provides a starting point for rational drug design for a class of targets that has gained increasing attention over the last years. ${ }^{[1 \mathrm{a}, \mathrm{b}, 22]}$

\section{Experimental Section}

Auoxo6 was synthesized as previously described. ${ }^{[23]}$ Tel26 ((AAA(GGGTTA) $)_{3}$ GGGAA) and wtTel26 ((TTAGGG) $)_{4}$ TT) DNA sequences were ordered in HPSF quality from Eurofins Genomics. Prior to usage, the DNAs were HPLC purified, desalted, and refolded in NMR-buffer by heating for $5 \mathrm{~min}$ to $95^{\circ} \mathrm{C}$ and subsequent slow cooling.

All NMR-titration spectra were recorded using a Bruker AVIII $600 \mathrm{MHz}$ spectrometer equipped with a cryoprobe at $298 \mathrm{~K}$ using $0.1 \mathrm{~mm}$ DNA in $25 \mathrm{~mm} \mathrm{KP}_{\mathrm{i}}, 70 \mathrm{~mm} \mathrm{KCl}, 0.03 \mathrm{~mm} \mathrm{DSS}, 10 \% \mathrm{D}_{2} \mathrm{O}$ at $\mathrm{pH}$ 7. Water suppression was achieved using a jump-return-echo pulse scheme. Auoxo6 was added directly from a DMSO stock solution; total DMSO concentration was never above $5 \%$. NOESY, ${ }^{[24]}$ $\operatorname{COSY}^{[24 b, d]}$ and $\mathrm{HSQC}^{[25]}$ spectra were recorded using either Bruker AVIII $600 \mathrm{MHz}$ or AV $700 \mathrm{MHz}$ spectrometers equipped with cryoprobes at $298 \mathrm{~K}$ using $1 \mathrm{~mm}$ DNA, $1.3 \mathrm{~mm}$ Auoxo6 in $25 \mathrm{~mm}$ $\mathrm{KP}_{\mathrm{i}}, 70 \mathrm{~mm} \mathrm{KCl}, 0.3 \mathrm{~mm}$ DSS, $7 \%$ [ $\left.\mathrm{D}_{6}\right] \mathrm{DMSO}$ at $\mathrm{pH} 7$ (either in $\mathrm{H}_{2} \mathrm{O}$ or $\mathrm{D}_{2} \mathrm{O}$ ). NMR spectra were recorded and processed using the software Topspin 3.2 (Bruker Biospin) and evaluated using the software Sparky 3.114. ${ }^{[2]}$ Assignment of free NMR-signals was taken from the literature. ${ }^{[13 a]}$

Structure calculation was performed essentially as described before ${ }^{[27]}$ with CNS1. $1^{[28]}$ using the ARIA $1.2^{[29]}$ setup and protocols, applying a nucleic acid force field and OPLS parameters for refinement in explicit water ${ }^{[30]}$ The topology and parameters for the Auoxo6 ligand were generated by hand and two potassium ions were included within the quadruplex tetrads for structure refinement. The chemical shifts were manually assigned and used for automated NOESY crosspeak assignment and calibration in ARIA. In total, 5 NOE peak list ( $50 \mathrm{~ms}, 100 \mathrm{~ms}$ in $\mathrm{H}_{2} \mathrm{O}, 50 \mathrm{~ms}, 100 \mathrm{~ms}, 150 \mathrm{~ms}$ in $\mathrm{D}_{2} \mathrm{O}$ ) were used. Furthermore, restraints were applied for hydrogen bonds, potassium coordination, base planarity, and the chi torsion angles of the G-quadruplex tetrads, sugar puckers were not restrained.

\section{Acknowledgements}

We thank Elke Stirnal for HPLC purification, Maria Dell for practical work and Irene Bessi for helpful discussions. This research was supported by the State of Hesse (LOEWE project: Synchembio and BMRZ), by the DFG (Cluster of Excellence: Macromolecular Complexes), and by the EU (H2020, iNEXT). Ente Cassa Risparmio Firenze was gratefully acknowledged for a grant to F.P. L.M. gratefully acknowledges Beneficentia Stiftung (Vaduz), ITT (Istituto Toscano Tumori), Fondazione Cassa Risparmio Firenze (CRF), AIRC (IG-16049) for financial support; thanks are also expressed to Prof. Maria Agostina Cinellu and Dr. Lara Massai, and to CIRCMSB.

\section{Conflict of interest}

The authors declare no conflict of interest.

Keywords: DNA-ligand complexes ·drug design . G-quadruplexes · NMR spectroscopy $\cdot$ structure elucidation 
How to cite: Angew. Chem. Int. Ed. 2017, 56, 7102-7106 Angew. Chem. 2017, 129, 7208-7212

[1] a) S. Balasubramanian, L. H. Hurley, S. Neidle, Nat. Rev. Drug Discovery 2011, 10, 261; b) S. Neidle, J. Med. Chem. 2016, 59 , 5987; c) D. Rhodes, H. J. Lipps, Nucleic Acids Res. 2015, 43, 8627.

[2] E. Henderson, C. C. Hardin, S. K. Walk, I. Tinoco, Jr., E. H. Blackburn, Cell 1987, 51, 899.

[3] J. L. Huppert, S. Balasubramanian, Nucleic Acids Res. 2007, 35 , 406.

[4] A. Siddiqui-Jain, C. L. Grand, D. J. Bearss, L. H. Hurley, Proc. Natl. Acad. Sci. USA 2002, 99, 11593.

[5] R. K. Moyzis, J. M. Buckingham, L. S. Cram, M. Dani, L. L. Deaven, M. D. Jones, J. Meyne, R. L. Ratliff, J. R. Wu, Proc Natl. Acad. Sci. USA 1988, 85, 6622.

[6] K. C. Healy, Oncol. Res. 1995, 7, 121.

[7] A. M. Zahler, J. R. Williamson, T. R. Cech, D. M. Prescott, Nature 1991, 350, 718.

[8] D. Hockemeyer, K. Collins, Nat. Struct. Mol. Biol. 2015, 22, 848.

[9] A. J. Zaug, E. R. Podell, T. R. Cech, Proc. Natl. Acad. Sci. USA 2005, 102, 10864.

[10] A. I. Karsisiotis, C. O'Kane, M. Webba da Silva, Methods 2013 , $64,28$.

[11] a) J. Dai, M. Carver, D. Yang, Biochimie 2008, 90, 1172; b) A. T. Phan, FEBS J. 2010, 277, 1107.

[12] a) R. Hansel, F. Lohr, S. Foldynova-Trantirkova, E. Bamberg, L. Trantirek, V. Dotsch, Nucleic Acids Res. 2011, 39, 5768; b) L. Petraccone, C. Spink, J. O. Trent, N. C. Garbett, C. S. Mekmaysy, C. Giancola, J. B. Chaires, J. Am. Chem. Soc. 2011, 133, 20951; c) R. Hänsel, F. Löhr, L. Trantirek, V. Dötsch, J. Am. Chem. Soc. 2013, 135, 2816.

[13] a) J. Dai, M. Carver, C. Punchihewa, R. A. Jones, D. Yang, Nucleic Acids Res. 2007, 35, 4927; b) J. Dai, C. Punchihewa, A. Ambrus, D. Chen, R. A. Jones, D. Yang, Nucleic Acids Res. 2007 35, 2440; c) A. T. Phan, V. Kuryavyi, K. N. Luu, D. J. Patel, Nucleic Acids Res. 2007, 35, 6517.

[14] a) J. Dai, M. Carver, L. H. Hurley, D. Yang, J. Am. Chem. Soc. 2011, 133, 17673; b) W. J. Chung, B. Heddi, M. Tera, K. Iida, K. Nagasawa, A. T. Phan, J. Am. Chem. Soc. 2013, 135, 13495.

[15] A. Casini, G. Kelter, C. Gabbiani, M. A. Cinellu, G. Minghetti, D. Fregona, H. H. Fiebig, L. Messori, J. Biol. Inorg. Chem. 2009, $14,1139$.

[16] A. Bindoli, M. P. Rigobello, G. Scutari, C. Gabbiani, A. Casini, L. Messori, Coord. Chem. Rev. 2009, 253, 1692.

[17] N. Micale, T. Schirmeister, R. Ettari, M. A. Cinellu, L. Maiore, M. Serratrice, C. Gabbiani, L. Massai, L. Messori, J. Inorg. Biochem. 2014, 141, 79 .
[18] P. Gratteri, L. Massai, E. Michelucci, R. Rigo, L. Messori, M. A. Cinellu, C. Musetti, C. Sissi, C. Bazzicalupi, Dalton Trans. 2015, 44, 3633.

[19] S. M. Haider, S. Neidle, G. N. Parkinson, Biochimie 2011, 93, 1239.

[20] C. Altona, M. Sundaralingam, J. Am. Chem. Soc. 1972, 94, 8205.

[21] a) A. Ponce-Salvatierra, K. Wawrzyniak-Turek, U. Steuerwald, C. Hobartner, V. Pena, Nature 2016, 529, 231; b) J. WirmerBartoschek, H. Schwalbe, Angew. Chem. Int. Ed. 2016, 55, 5376; Angew. Chem. 2016, 128, 5462.

[22] a) I. Bessi, C. Bazzicalupi, C. Richter, H. R. Jonker, K. Saxena, C. Sissi, M. Chioccioli, S. Bianco, A. R. Bilia, H. Schwalbe, P. Gratteri, ACS Chem. Biol. 2012, 7, 1109; b) D. Panda, M. Debnath, S. Mandal, I. Bessi, H. Schwalbe, J. Dash, Sci. Rep. 2015, 5, 13183; c) Y. Pavan Kumar, P. Saha, D. Saha, I. Bessi, H. Schwalbe, S. Chowdhury, J. Dash, ChemBioChem 2016, 17, 388.

[23] a) A. Casini, M. A. Cinellu, G. Minghetti, C. Gabbiani, M. Coronnello, E. Mini, L. Messori, J. Med. Chem. 2006, 49, 5524; b) M. A. Cinellu, A. Zucca, S. Stoccoro, G. Minghetti, M. Manassero, M. Sansoni, J. Chem. Soc. Dalton Trans. 1996, 4217.

[24] a) G. Lippens, C. Dhalluin, J. M. Wieruszeski, J. Biomol. NMR 1995, 5, 327; b) M. Piotto, V. Saudek, V. Sklenar, J. Biomol. NMR 1992, 2, 661; c) V. Sklenar, J. Magn. Reson. Ser. A 1995, 114, 132; d) V. Sklenar, M. Piotto, R. Leppik, V. Saudek, J. Magn. Reson. Ser. A 1993, 102, 241.

[25] a) L. E. Kay, P. Keifer, T. Saarinen, J. Am. Chem. Soc. 1992, 114, 10663 ; b) A. G. Palmer, J. Cavanagh, P. E. Wright, M. Rance, J. Magn. Reson. 1991, 93, 151; c) J. Schleucher, M. Schwendinger, M. Sattler, P. Schmidt, O. Schedletzky, S. J. Glaser, O. W. Sorensen, C. Griesinger, J. Biomol. NMR 1994, 4, 301.

[26] T. D. K. Goddard, D. G., University of California, San Francisco.

[27] J. Thevarpadam, I. Bessi, O. Binas, D. P. Goncalves, C. Slavov, H. R. Jonker, C. Richter, J. Wachtveitl, H. Schwalbe, A. Heckel, Angew. Chem. Int. Ed. 2016, 55, 2738; Angew. Chem. 2016, 128, 2788.

[28] A. T. Brünger, P. D. Adams, G. M. Clore, W. L. DeLano, P. Gros, R. W. Grosse-Kunstleve, J. S. Jiang, J. Kuszewski, M. Nilges, N. S. Pannu, R. J. Read, L. M. Rice, T. Simonson, G. L. Warren, Acta Crystallogr. Sect. D 1998, 54, 905.

[29] J. P. Linge, S. I. O'Donoghue, M. Nilges, Methods Enzymol. 2001, 339, 71 .

[30] S. Nozinovic, B. Furtig, H. R. Jonker, C. Richter, H. Schwalbe, Nucleic Acids Res. 2010, 38, 683.

Manuscript received: February 27, 2017

Revised manuscript received: March 31, 2017

Version of record online: May 15, 2017 